\title{
Original
}

\section{Characteristics and Chemosensitivity of Hepatoblastoma Xenograft}

\author{
Yuko Tsunoda, Miki Shibusawa*, Yoshihiro Kabasawa, \\ Jun-ichi Suzuki, Akira Gomi, Masashi Yatsuzuka \\ and Takao OKAмASTU
}

\begin{abstract}
A 6-month-old girl presented with an abdominal mass and high serum levels of $\alpha$-fetoprotein. Computed tomography showed an extremely large tumor of the liver, which was diagnosed with open biopsy as a well-differentiated hepatoblastoma. Left hepatic lobectomy was performed after regional chemotherapy, including cisplatin, which was effective. The biopsy specimen was transplanted to a nude mouse, and a xenograft was successfully established. The xenograft maintained the characteristics of the original tumor with regard to cell structure and the high serum level of $\alpha$ fetoprotein in both hosts. A part of the xenograft was subjected to methylthiotetrazole assay. Cisplatin was most effective, as it had been clinically. Xenografts may be useful for determining the chemosensitivity of hepatoblastoma.
\end{abstract}

Key words : hepatoblastoma, xenograft, chemosensitivity

\section{Introduction}

Human tumor xenografts have been widely used for chemosensitivity testing because they maintain the characteristics of the original tumor. Such characteristics of juvenile neuroblastoma as oncogenes and drug sensitivity have been investigated with cell lines and xenografts $^{1,2)}$. Although hepatoblastoma is known to produce $\alpha$-fetoprotein (AFP), its characteristics are not well described. We previously reported a non-AFP-producing hepatoblastoma cell line ${ }^{3)}$. In the present study, an AFP-producing hepatoblastoma xenograft was investigated.

\section{Clinical history}

A girl was born at 41 weeks' gestation with a weight of $3.26 \mathrm{~kg}$. At 6 months of age, she was noted to have an abdominal mass in the right upper quadrant and was admitted to our hospital. Computed tomography (CT) showed an extremely large tumor of the left lobe of the liver. The serum level of AFP was 3,867,000 ng/ml. An open biopsy of the liver revealed a well-differentiated hepatoblastoma. Before surgery, regional chemotherapy consisting of cisplatin (CDDP) and THP-adriamycin (THP-Ad) was administered via the left hepatic artery. Eight weeks after treatment, CT revealed that the tumor had decreased in size and the concentration of serum AFP had decreased to 28,900 ng/ml. Left hepatic

Department of Pediatric Surgery, Showa University School of Medicine, 1-5-8, Hatanodai, Shinagawa-ku, Tokyo 142-8666, Japan.

* Second Department of Surgery, Showa University School of Medicine. 


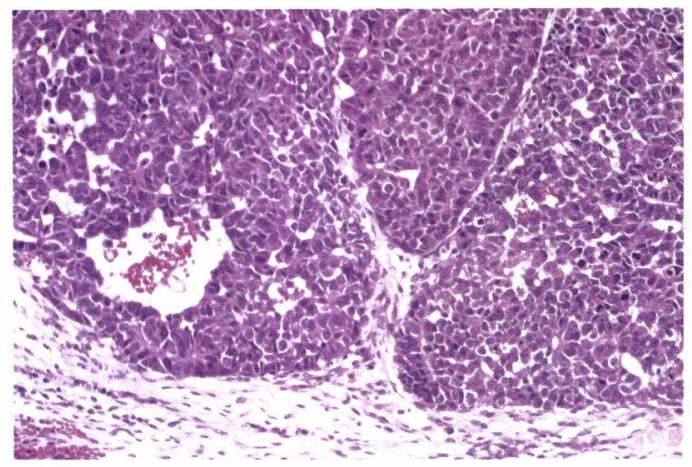

Fig. 1. Microscopic finding of the xenograft in a nude mouse showing a well-differentiated hepatoblastoma $(\mathrm{H}-\mathrm{E}, \times 40)$.

lobectomy was performed with excision of the tumor. Seven months after the operation, the serum AFP level was less than $3 \mathrm{ng} / \mathrm{ml}$ and no recurrence of tumor was detected on CT. The patient has been well in the 4 years since the operation.

\section{Characteristics of hepatoblastoma xenograft}

The liver biopsy specimen was transplanted to the back of a BALB/c nude mouse. One month later, a tumor had formed subcutaneously. It was removed, and blood samples were obtained at necropsy. Histologically, the cell structure of the tumor was similar to that of the primary tumor (Fig. 1). The tumor's doubling time was 6 days. The serum concentration of AFP in the nude mouse was $98,300 \mathrm{ng} / \mathrm{ml}$, and the percentage of Con A-non-bound AFP was $8 \%$. The mode of the chromosome number was 50 , and the phenotype was $50, \mathrm{XX},+\operatorname{der}(1 \mathrm{q} 7 \mathrm{p})+2+8+20$. The DNA of the xenograft was aneuploid. N-myc and c-myc were not detected with high amplication. The avidin-biotinperoxidase complex method was used for immunohistochemical studies for AFP, $\alpha$-1-antit rypsin and cytokeratin (Fig. 2). One-third of the carcinoma cells were positive for epidermal growth factor (Fig. 3).

\section{Chemosensitivity test (MTT assay)}

The assay is dependent on the cellular reduction of methylthiotetrazole (MTT, Sigma Chemical Co., St. Louis, MO) by the mitochondrial dehydrogenese of viable cells to a dark blue formazan product that can be measured spectrophotometrically ${ }^{4}$.

A single-cell suspension was obtained by trypsinization of cells through a $200 \mu \mathrm{m} / \mathrm{s}$ stainless-steel mesh after the nude mouse tumor was minced, with cell counts performed with the dye exclusion test. Cells at a concentration of $5 \times 10^{5} / \mathrm{ml}$ in RPMI 1640 supplemented with $20 \%$ fetal calf serum were plated onto 96-well microtiter plates at a volume of $100 \mu \mathrm{l} /$ well. 5-Fluorouracil (5-FU), THP-Ad, and CDDP were used. Drugs dissolved in RPMI 1640 were also added at twice the final concentration at a volume of $100 \mu \mathrm{l} /$ well. The plates were incubated for 48 hours at $37{ }^{\circ} \mathrm{C}$ in a humidified atmosphere containing $95 \%$ air $/ 5 \% \mathrm{CO}_{2}$. Using the procedure described by Carmichael et al ${ }^{5)}$ with MTT solution and DMSO (Sigma), the plates were read on a two-wave length microplate photometer (MTP-120, Corona Electric Co., Ltd. Tokyo, Japan). All drug concentrations 


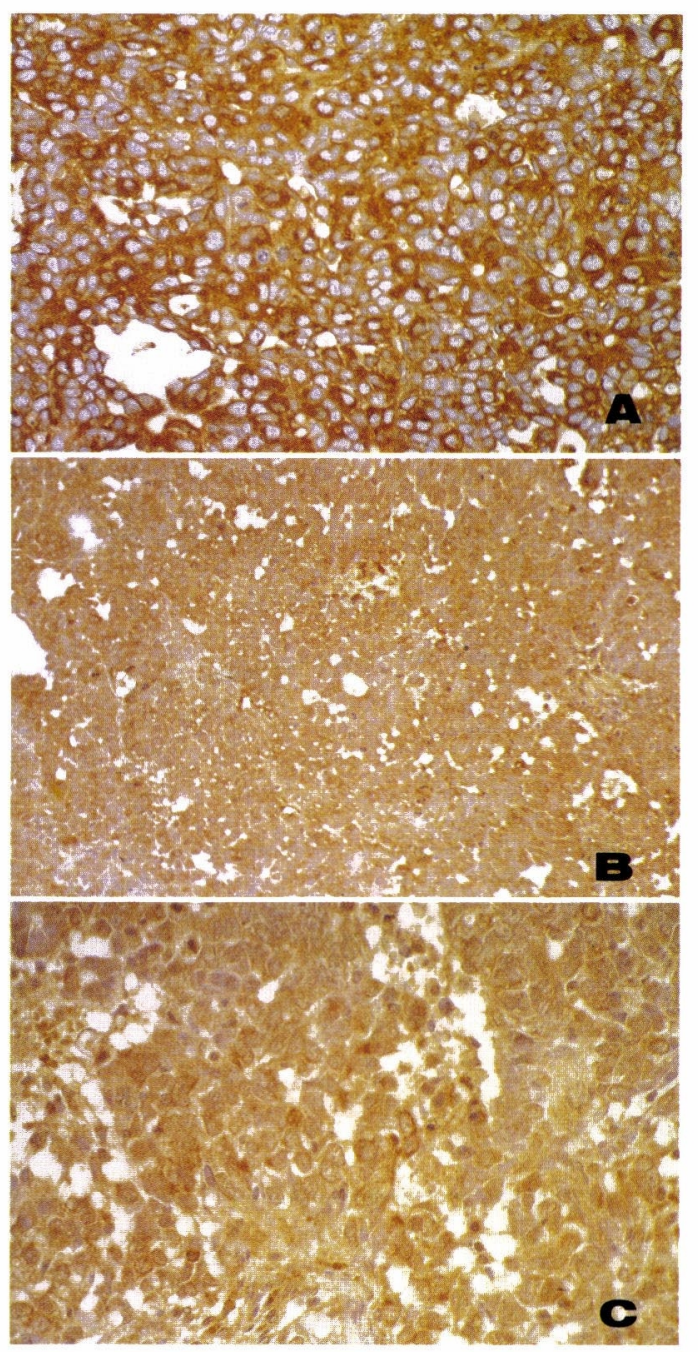

Fig. 2. Well-differentiated hepatoblastoma in a xenograft showing moderate to intense immunohistochemical staining.
A. $\operatorname{AFP}(\times 100)$,
B. $\alpha$-1-antitrypsin $(\times 40)$,
C. cytokeratin $(\times 100)$.

were tested in triplicate wells. The inhibition rate at each concentration was calculated as follows :

inhibition rate $(\%)=(1-t / c) \times 100$

$t$ : mean absorbance of the treated wells

$c$ : mean absorbance of the control wells

Figure 4 shows the inhibition rates of 5-FU, THP-Ad, and CDDP against hepatoblastoma xenograft cells. The inhibition rates of 5-FU and THP-Ad were almost unchanged at less than $40 \%$, whereas those of CDDP were higher than $60 \%$ within the range of doses tested. 


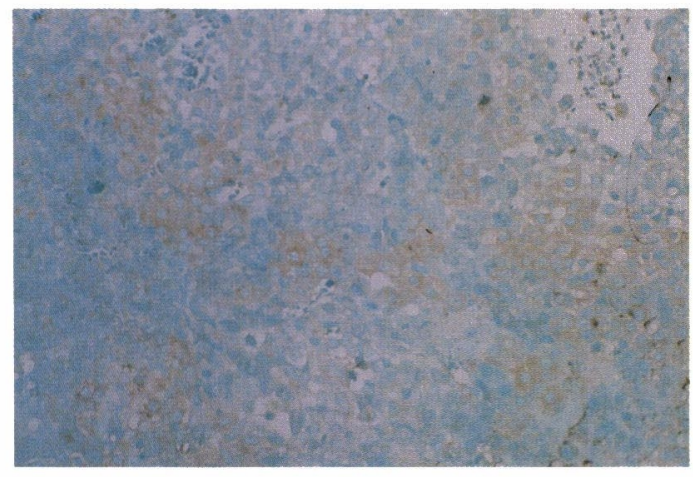

Fig. 3. Xenograft tissue with moderate epidermal growth factor expression $(\times 100)$.

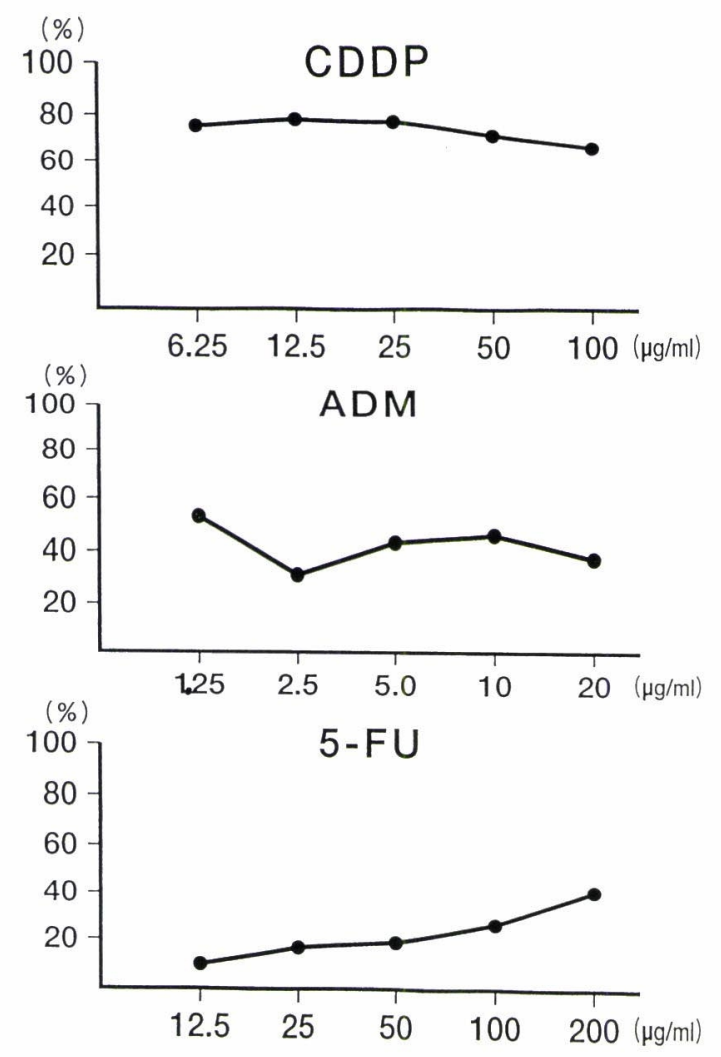

Fig. 4. Effects of 5-fluorouracil, THP-adriamycin and cisplatin against xenograft cells with the MTT assay.

\section{Discussion}

Hepatoblastoma is one of the most common malignant solid tumors in childhood and is known to produce high serum levels of AFP. Because few studies of hepatoblastoma xenografts have been performed, their biochemical characterisitics are not known in detail. 
In the present case, the xenograft closely resembled the original tumor because of the high serum levels of AFP in both hosts and the similarity of cell structure. Chromosome analysis showed duplication of chromosomes 2,8 and 20 , in addition to 1q7p. Amplification of $7 p$ has been reported to increase production of epidermal growth factor in tumors 6) Staining of the xenograft for epidermal growth factor was positive in the present study, suggesting that production may be enhanced by amplification of $7 \mathrm{p}$.

Some reports have suggested a relationship between p53 and chemosensitivity. In malignant glioma, the WAF1/CIP1 gene was induced in p53-dependent apoptosis of U87-MG glioma cells by $\mathrm{CDDP}^{7)}$. However, in small cell lung carsinoma cells, the effect of wild-type p53 gene transfer was stronger than that of the mutant p53 gene in sensitizing tumor cells to CDDP in and correlated with the induction of apoptosis ${ }^{8)}$.

We previously investigated mutations of the p53 gene in 38 hepatoblastomas and in two hepatoblastoma cell lines with polymerase chain reaction-single stand conformation polymorphism ${ }^{9}$. Mutations of the p53 gene were detected only in the cell line of an anaplastic hepatoblastoma against which chemotherapy had been ineffective. However, no abnormalities of the p53 gene were found in other hepatoblastomas, including the present tumor. Because the present tumor was highly sensitive to CDDP, mutant p53-independent apoptosis of the tumor cells by CDDP was suggested.

Regarding the effect of anticancer drugs against hepatoblastoma, combination chemotherapy using more than two antitumor drugs has been reported to be successful ${ }^{10,11)}$, but the effects of individual drugs have not, to our knowledge, been reported in the literature.

The MTT assay is used as a chemosensitivity test in vitro and is reported to have several advantage with respect to speed and quantitation ${ }^{12)}$. In the present case, the biopsy specimen was too small for the antitumor effect of anticancer drugs to be examined. The biopsy specimen was subcutaneously transplanted to the back of a nude mouse. When the nude mouse tumor was $10 \mathrm{~mm}$ in diameter, the tumor was subjected to MTT assay. The drugs for the MTT assay were 5-FU, THP-Ad, and CDDP, because these drugs were used in the Japanese Pilot Study II $^{18)}$. The results of this assay were consistent with the clinical reduction in the liver tumor's size. In particular, CDDP was most effective in this assay.

Sakamoto et al $^{14)}$ reported that because xenografts have the same chemosensitivity as the original tumor, the effect of chemotherapy can be predicted with the MTT assay. From this point of view, the present xenograft and the MTT assay may be useful for determining the effectiveness of newly developed antineoplastic agents against hepatoblastoma.

\section{References}

1) Horie H, Sugaya Y, Harigaya K, Etoh T and Maie M : Expression of cellular adhesion molecules (NCAM and CD44) in neuroblastoma. $J$ Jpn Soc Pediatr Surg 32 : 790-794 (1996) (in Japanese)

2) Hemmi H, Yamada K, Kato M, Yang HW, Iwasaki I, Tsuchida Y and Shimatake H : Coexpression of N-myc, c-myc, and L-myc oncogenes in human established cell lines. Jpn J Pediatr Surg 29: 406-411 (1997) (in Japanese)

3) Tsunoda Y, Shibusawa M, Okamatsu, Yatsuzuka M and Iijima T: Non-alpha-fetoprotein-producing anaplastic hepatoblastoma cell line. In Vitro Cell Dev Biol Animal 32 : 194-196 (1996)

4) Mosmann T: Rapid colorimetric assay for cellular growth and survival: Application to proliferation and cytotoxicity assays. J Immunol Methods 65 : 55-63 (1983)

5) Carmichael J, DeGraff WG, Gazdar AF, Minna JD and Mitchell JB: Evaluation of a tetrazolium-based semiautomated colorimetric assay: Assessment of chemosensitivity testing. Cancer Res 47 : 936-942 (1987)

6) Tsuda H and Hirohashi S : Alterations of oncogenes in human cancers. Gann To Kagakuryouhou 20:977-983 (1993) (in Japanese) 
7) Kondo S, Barna BP, Kondo Y, Tanaka Y, Casey G, Liu J, Morimura T, Kaakaji R, Peterson JW, Werbel B and Barnett GH: WAF1/CIp1 increases the susceptibility of 'p53' non-functional malignant glioma cells to 'cisplatin' -induced apoptosis. Oncogene 13 : 1279-1285 (1996)

8) Gjerset RA, Turia ST, Sobol RE, Scalise JJ, Mercola D, Collins H and Hopkins PJ : Use of wild-type 'p53' to achieve complete treatment sensitization of tumor cells expressing endogenous mutant 'p53' . Mol Carcinog 14 : 275-285 (1995)

9) Ohnishi H, Kawamura M, Hanada R, Kaneko Y, Tsunoda Y, Hongo T, Bessho F, Yokomori K and Hayashi $\mathrm{Y}$ : Infrequent mutations of the TP53 gene and no amplification of the MDM2 gene in hepatoblastomas. Genes Chromosomes Cancer 15 : 187-190 (1996)

10) Pierro A, Langevin AM, Filler RM, Liu P, Phillips MJ and Greenberg ML: Preoperative chemotherapy in 'unresectable' hepatoblastoma. J Pediatr Surg 24 : 24-29 (1989)

11) Reynolds M, Douglass EC, Finegold M, Cantor A and Glicksman A : Chemotherapy can convert unresectable hepatoblastoma. J Pediatr Surg 27 : 1080-1084 (1992)

12) Shimoyama $Y$, Kubota T, Watanabe M, Ishibiki $\mathrm{K}$ and Abe $\mathrm{O}$ : Predictability of in vivo chemosensitivity by in vitro MTT assay with reference to clonogenic assay. J Surg Oncol 41 : 12-18 (1989)

13) Uchino $\mathbf{J}$ : Liver tumor in childhood: prospective chemotherapeutic controlled randomized trial in Japan. $J$ Clin Exp Med 156: 122 (1991)

14) Sakamoto Y, Fujita M, Goi M, Tsukamoto F, Sugimoto T, Okuyama Y, Rhi M, Kusuyama $T$ and Fujita F : Accordance of the chemosensitivity between clinical specimens and their xenografts in nude mice by SDI test and the value of in vivo chemosensitivity test using nude mice. Gann To Kagakuryouhou 20: 447-454 (1993) (in Japanese)

[Received August 12, 1997 : Accepted September 17, 1997] 